\title{
Uso e necessidade de prótese dentária em idosos nordestinos: avanços, perspectivas e desafios
}

Needs for dental prosthesis and their use in northeastern elderly: advances, perspectives and challenges

Uso y necesidad de prótesis dentales en ancianos del Nordeste: avances, perspectivas y desafíos

Iúska Cyntia Mariz GALVÃo

Graduanda em Odontologia pela Universidade Estadual da Paraíba- UEPB, 58401- 490 Campina Grande- PB, Brasil

Allyson Martim Medeiros LIRA

Graduando em Odontologia pela Universidade Estadual da Paraíba- UEPB, 58401- 490 Campina Grande- PB, Brasil

Diego Almeida MAGALHÃES

Graduando em Odontologia pela Universidade Estadual da Paraíba- UEPB, 58401- 490 Campina Grande- PB, Brasil Marcelo Marques GOUVEIA FILHO

Graduando em Odontologia pela Universidade Estadual da Paraíba- UEPB, 58401- 490 Campina Grande- PB, Brasil Sandryenne Rayanne Barbosa Gois SANTOS Graduanda em Odontologia pela Universidade Estadual da Paraíba- UEPB, 58401- 490 Campina Grande- PB, Brasil Maria das Graças Barbosa da SILVA Doutoranda em Odontologia pela Universidade Estadual da Paraíba-UEPB, 58401-490 Campina Grande- PB, Brasil Maria Helena Chaves de Vasconcelos CATÃO Professora Titular, Universidade Estadual da Paraíba- UEPB, 58401- 490 Campina Grande- PB, Brasil https://orcid.org/0000-0001-7681-3225

\section{Resumo}

Segundo dados de 2018, colhidos da agência de notícias do Instituto Brasileiro de Geografia e Estatística (IBGE), o número de idosos havia crescido $18 \%$ em cinco anos e ultrapassava 30 milhões em 2017. De forma a ratificar esse processo de envelhecimento populacional, o Ministério da Saúde inteirou que em 2030 o número de idosos ultrapassará o total de crianças, o que gera uma maior necessidade de atenção e sensibilidade para com essa imensa parcela da população, que no passado foi submetida a uma prática odontológica mutiladora atrelada à iniqüidade no acesso aos serviços de saúde bucal, acarretando em alto índice de edentulismo, ainda persistente após a implantação dos Laboratórios Regionais de Prótese Dentária (LRPDs), incorporados pela Política Nacional de Saúde Bucal. Desse modo, o presente artigo objetiva uma análise dos avanços e perspectivas do uso e inópia de prótese dentária em idosos nordestinos, a fim de evidenciar a importância de um alcance ainda maior da Odontologia reabilitadora para acréscimo na qualidade de vida dos idosos brasileiros.

Descritores: Prótese Dentária; Inquéritos de Saúde Bucal; Odontologia Geriátrica.

\section{Abstract}

According to information from 2018, collected from the news agency of the Brazilian Institute of Geography and Statistics (IBGE), the number of elderly people had grown $18 \%$ in five years and exceeded 30 million in 2017 . In order to ratify this process of population aging, the Ministry of Health found out that in 2030 the number of elderly people will exceed the total number of children, which creates a greater need for attention and sensitivity towards this immense portion of the population, which in the past has been subjected to a mutilating dental practice linked to inequity in access to oral health services, resulting in a high rate of edentulism, which is still persistent after the implantation of the Regional Dental Prosthesis Laboratories (LRPDs), incorporated by the National Oral Health Policy. Thus, the present article aims to analyze the advances and perspectives of the use and inopia of dental prosthesis in northeastern elderly, in order to highlight the importance of an even greater reach of rehabilitation dentistry to increase the quality of life of elderly Brazilians.

Descriptors: Dental Prosthesis; Dental Health Surveys; Geriatric Dentistry.

\section{Resumen}

Según datos de 2018, recogidos de la agencia de noticias del Instituto Brasileño de Geografía y Estadística (IBGE), el número de ancianos había crecido un $18 \%$ en cinco años y superó los 30 millones en 2017. Para confirmar este proceso de población Envejecimiento, el Ministerio de Salud informó que en el 2030 el número de ancianos superará al total de niños, lo que genera una mayor necesidad de atención y sensibilidad a esta inmensa porción de la población, que en el pasado fue sometida a una mutilación dental. práctica ligada a la inequidad en el acceso a los servicios de salud bucal, resultando en una alta tasa de edentulismo, que aún persiste luego de la implementación de los Laboratorios Regionales de Prótesis Dentales (LRPD), incorporados a la Política Nacional de Salud Bucal. Así, este artículo tiene como objetivo analizar los avances y perspectivas del uso y la inopia de prótesis dentales en ancianos del Nordeste, con el fin de resaltar la importancia de un alcance aún mayor de la odontología rehabilitadora para incrementar la calidad de vida de los ancianos brasileños.

Descriptores: Prótesis Dental; Encuestas de Salud Bucal; Odontología Geriátrica.

INTRODUÇÃO

O aumento do número da população idosa indica uma realidade demográfica expressiva no Brasil e no mundo'. Pesquisas sociodemográficas de cunho populacional têm indicado que a quantidade de idosos, em números relativos e absolutos, está crescendo mais rapidamente do que qualquer outra faixa etária ${ }^{1,2}$.

Esse novo perfil populacional, além de evidenciar marcada alteração na estrutura social do país, confere uma maior probabilidade de riscos e desenvolvimento de incapacidades associadas ao envelhecimento, o que demarca um novo perfil de morbimortalidade com reflexo direto no aumento dos custos com assistência à saúde ${ }^{3,4}$.
Ao mesmo tempo em que decrescem os níveis de taxas de mortalidade e natalidade, crescem a proporção de idosos na população. No Brasil, a proporção de pessoas com 60 ou mais anos de idosos na população, na década de 90 era de $7,5 \%$, subindo para $10,8 \%$ em 2010. O indicativo para o ano de 2015 foi de aproximadamente $13 \%$ de idosos na população total e a projeção para 2030 é de $18,05 \%$ de idosos na população brasileira ${ }^{5}$.

No Brasil, a datar do início do desenvolvimento do Sistema Único de Saúde (SUS), vários avanços na organização da atenção primária à saúde (APS) foram reconhecidos $^{6,7}$. $\mathrm{Na}$ área odontológica, as diretrizes da Política Nacional de Saúde Bucal 
publicada em 2004, por meio do Programa Brasil Sorridente, tinham como objetivo interpor os procedimentos relacionados com a fase clínica da instalação de próteses dentárias na APS, tal como garantir a implantação dos laboratórios regionais de prótese dentária ${ }^{7,8}$.

Através dos dados do levantamento nacional de saúde bucal (Projeto SB Brasil 2010 - Pesquisa Nacional de Saúde Bucal), notou-se que cerca de $92,7 \%$ na faixa etária entre 65 a 74 anos ainda necessitavam de prótese dentária $^{8}$. Dito isto, estudos que avaliam as necessidades da população idosa com relação à saúde bucal e os fatores associados a estas necessidades podem ser úteis para 0 fornecimento de subsídios para o planejamento dos serviços de caráter reabilitador ${ }^{9-12}$.

Destarte, este estudo teve como objetivo analisar a prevalência de uso e necessidade de prótese dentária em idosos (65 a 74 anos) na região Nordeste brasileira, a partir de dados do Projeto SB Brasil 2003 e 2010.

MATERIAL E MÉTODO

Trata-se de um estudo transversal, realizado com dados secundários dos inquéritos sobre as Condições de Saúde Bucal da População Brasileira, denominado SB Brasil, dos anos de 2003 e 2010. Esses levantamentos epidemiológicos em saúde bucal foram desenvolvidos seguindo metodologia sugerida pelo Ministério da Saúde.

Foram pesquisadas as variáveis clínicas - uso e necessidade de prótese dentária superior e inferior do levantamento SB Brasil 2003 e 2010 - na população com faixa etária entre 65 a 74 anos, da região Nordeste do Brasil, com uma amostra total de 1.443 idosos em 2003 e 2.269 em 2010.

O uso e a necessidade de prótese são investigados baseando-se em índice desenvolvido pela própria OMS (Organização Mundial de Saúde), que determina a necessidade considerando as perdas dentárias existentes nos arcos superior e inferior, sendo o uso computado pela presença de um ou mais tipos de prótese em um ou nos dois arcos. Depois de obtidos os dados, foram analisados os percentuais na distribuição de uso e necessidade de prótese na população de estudo, existentes nas tabelas e variáveis citadas. Não houve necessidade de submissão ao Comitê de Ética Envolvendo Seres Humanos, uma vez que se analisaram os resultados já divulgados do Projeto SB Brasil. RESULTADOS

A Tabela 1 mostra o uso de prótese dentária de acordo com o tipo de prótese, entre idosos na faixa etária de 65 a 74 anos de idade, na região Nordeste do país, analisando os anos de 2003 e 2010.

No levantamento epidemiológico SB Brasil 2003 verificou-se que $51,8 \%$ dos idosos estavam usando algum tipo de prótese na arcada superior ( $n=749$ ) e $24,04 \%$ usam prótese na arcada inferior $(n=457)$. Nos dados adquiridos pelo SB Brasil 2010 verificou-se que $68,6 \%$ dos idosos estavam usando algum tipo de prótese na arcada superior $(n=1.556)$ e $44,5 \%$ usam prótese na arcada inferior $(n=1.009)$. Ou seja, houve um aumento considerável de quase duas vezes no número de idosos com algum tipo de prótese na arcada inferior (Tabela 1).

Tabela 1. Uso de prótese dentária, segundo o tipo de prótese, entre idosos na faixa etária de 65-74 anos, na região Nordeste

\begin{tabular}{|l|c|c|c|c|c|c|c|c|}
\hline \multirow{2}{*}{} & \multicolumn{4}{|c|}{2003} & \multicolumn{4}{c|}{2010} \\
\cline { 2 - 9 } & Superior & Inferior & Superior & Inferior \\
\cline { 2 - 9 } & $\mathrm{n}$ & $\%$ & $\mathrm{n}$ & $\%$ & $\mathrm{n}$ & $\%$ & $\mathrm{n}$ & $\%$ \\
\hline Não Usa & 695 & 48,13 & 987 & 51,92 & $* *$ & 31,4 & ${ }^{* *}$ & 55,5 \\
\hline Usa & 749 & 51,87 & 457 & 24,04 & ${ }^{* *}$ & 68,6 & $* *$ & 44,5 \\
\hline Total & 1.444 & 100 & 1.444 & 75,96 & 2.269 & 100 & 2.269 & 100 \\
\hline Fonte: Dados da Pesquisa
\end{tabular}

Quanto à necessidade de prótese (Tabela 2), os dados apresentando pelo SB Brasil 2003 diferenciam-se em prótese superior e prótese inferior, $46,3 \%(n=669)$ e $66,6 \%$ $(n=961)$, dos idosos avaliados necessitavam de próteses superiores e inferiores respectivamente. A maior necessidade avaliada entre os parâmetros registrados foi à necessidade de prótese total, considerando ambas as arcadas $(21,2 \%$ arcada superior $26,77 \%$ para arcada inferior).

Tabela 2. Necessidade de prótese dentária, segundo o tipo de prótese, entre idosos na faixa etária de 65-74 anos, na região Nordeste

\begin{tabular}{|c|c|c|c|c|c|c|}
\hline & \multicolumn{4}{|c|}{2003} & \multicolumn{2}{|c|}{2010} \\
\hline & \multicolumn{2}{|c|}{ Superior } & \multicolumn{2}{|c|}{ Inferior } & & \\
\hline & $\mathrm{n}$ & $\%$ & $\mathrm{n}$ & $\%$ & $\mathrm{n}$ & $\%$ \\
\hline Não necessita & 774 & 53,64 & 481 & 33,36 & *** & 3,9 \\
\hline Necessita & 669 & 46,36 & 961 & 66,64 & $* *$ & 96,1 \\
\hline Total & 1.443 & 100 & 1.442 & 100 & 1.303 & 100 \\
\hline
\end{tabular}

No levantamento realizado em 2010 não houve diferenciação entre a necessidade de prótese em superior ou inferior, referindo-se apenas às variáveis observadas: Parcial 1 maxilar e Total 1 maxilar, e apresentou dados agrupados de ambas as arcadas pelo tipos de prótese: Parcial 2 maxilar, Parcial + Total e Total 2 maxilar. Quanto à necessidade de prótese, houve maior valor para a variável Parcial 1 maxilar (29\%) e menor valor para a variável Parcial+Total (6,7\%). Observou-se, com os dados deste levantamento, que $96,1 \%$ $(n=1.252)$ dos idosos necessitavam de uso de próteses.

$\mathrm{Na}$ faixa etária de 65 a 74 anos, apenas 23,5\% não usava algum tipo de prótese dentária 
superior, sendo o maior percentual $(31,4 \%)$ na região Nordeste. A porcentagem de usuários de prótese total foi de $63,1 \%$ para o Brasil, variando de $65,3 \%$ na região Sul a 56,1\% na região Nordeste. Na faixa etária de 65 a 74 anos a proporção de indivíduos que não usava prótese inferior é de $46,1 \%$, sendo maior nas regiões Norte e Nordeste (55\%).

A porcentagem de usuários de prótese total foi de $37,5 \%$ para o Brasil, estando a maioria na região Sul $(40,4 \%)$ e a menor porcentagem na região Nordeste $(30,8 \%)$. Um quinto das pessoas tinha necessidade de prótese parcial para dois maxilares, sendo que a maior necessidade estava na região Nordeste (26,0\%).

DISCUSSÃO

Trata-se de um estudo transversal, realizado com dados secundários dos inquéritos sobre as Condições de Saúde Bucal da População Brasileira, dos anos de 2003 e 2010. Quanto ao uso e necessidade de prótese entre idosos brasileiros na faixa etária de 65 a 74 anos, na região do Nordeste brasileiro, o principal resultado deste estudo é observar a necessidade e o de prótese dentária, na região do Nordeste brasileiro, segundo dados do SB 2003 e 2010.

Nota-se que a preponderância e a distribuição das perdas dentárias variam significantemente de acordo com o grau de desenvolvimento dos países, sendo esta variação correlacionada a uma combinação de fatores intrínsecos, culturais e socioeconômicos ${ }^{13,14}$.

O uso de prótese foi maior no arco superior e as próteses dentárias mais utilizadas foram à prótese total e prótese parcial removível que substituem todos os elementos e mais de um elemento, respectivamente. $O$ desgaste ou perda dentária em idosos está relacionado a fatores socioeconômicos, condições de saúde e qualidade de vida ${ }^{15-17}$. Relacionado aos indicadores já ressaltados, a idade avançada dos idosos se mostra um fator relevante, que pode ser associado aos agravos no perfil de saúde doença do idoso e que, quando agregada à ideia de baixa renda, advindas de aposentadoria ou da sua inexistência, influência negativamente sua condição de saúde ${ }^{18}$. A situação socioeconômica na faixa etária idosa impacta de forma negativa suas relações sociais, bem como os hábitos de vida e o acesso aos serviços de saúde pública, fatores que em conjunto contribuem de forma negativa a saúde deles ${ }^{19}$.
A prática
de
higiene
bucal pode

influenciar a presença de placa bacteriana, podendo haver correlação entre frequência de escovação com número de dentes. A literatura tem mostrado que a higiene bucal precária e a dificuldade em realizar controle mecânico da placa em níveis adequados entre idosos, influência diretamente na saúde bucal do idoso, e pode assim corroborar para doenças oportunistas $^{20,21}$.

Segundo Marcus et al. ${ }^{22}$ e Imholz et al. ${ }^{23}$, concordando com os achados encontrados nesta pesquisa, observa-se uma piora da saúde bucal dos idosos investigados. Poucos permaneceram com a mesma quantidade de dentes, aumentando principalmente o número de usuários de prótese inferior. No tocante aos fatores associados ao uso e necessidade de prótese, após o ajuste por medidas de saúde geral e uso de serviços odontológicos, a presença de dentição funcional permaneceu significativamente associada às medidas socioeconômicas e demográficas, confirmando a presença de iniquidades presentes na sociedade e observadas por diversos outros estudos desenvolvidos ${ }^{17,24,25}$.

Independente da medida utilizada, número de dentes perdidos, edentulismo ou dentição funcional, a chance desse desfecho tem sido significativamente maior entre os indivíduos menos escolarizados ${ }^{26,27}$ sendo possível afirmar que há um grau de variação social associada a perda dentária ${ }^{28} \cdot \mathrm{Geib}^{29}$ e Soares et al. $^{30}$ ao estudarem o processo de alfabetização na faixa etária idosa no Brasil, destacaram, que o mesmo reflete as políticas nacionais e educacionais a distância, na qual o ensino fundamental repercutia o processo de exclusão educacional,o que justificaria o índice expressivo de analfabetismo em idosos no Brasil, principalmente em regiões interioranas e de difícil acesso. De acordo com o PNAD (2015) o índice de analfabetismo no segmento etário de 60 anos ou mais, no Brasil, era de aproximadamente $23,1 \%$.

De acordo com Dutra e Sanchez ${ }^{31}$, a faixa etária que compreende os idosos da atualidade traz consigo a herança de um modelo de saúde assistencial centrado na doença, com práticas curativas e mutiladoras. $\mathrm{O}$ cenário atual de saúde da população idosa é bastante precário e, em relação à saúde bucal, nota-se a grande quantidade de problemas bucais, principalmente relacionado à perda dentária e uso e necessidade de próteses, sendo que este último demanda serviços reabilitadores para a população. Porém, o serviço protético fornecido pelo Estado é recente, advindo da Política Nacional de Saúde 
Bucal de 2004, por meio de implementação dos Laboratórios Regionais de Prótese Dentária.

CONCLUSÃO

Pode-se concluir que o uso de prótese dentária ainda é muito baixo em ambos os inquéritos observados, dado o grande número de edentulismo existente entre os idosos das pesquisas.

Os dois estudos epidemiológicos evidenciam a necessidade que as pessoas desse grupo etário têm de serem reabilitadas por meio de uso de próteses parciais ou totais. Tais achados apontam que há uma necessidade de redirecionamento nas políticas públicas a fim de que a assistência, tão rara a essa população, seja balizada por ações que atinjam veementemente a cultura da naturalização da perda dentária/protesismo.

\section{REFERÊNCIAS}

1. Sander M, Oxlund B, Jespersen A, Krasnik A, Mortensen EL, Westendorp RGJ, et al. The challenges of human population ageing. Age Ageing. 2015;44(2):185-7.

2. Pinto RBR, Bastos LC.Abordagem das pesquisas em epidemiologia aplicada à gerontologia no Brasil: revisão da literatura em periódicos, entre 1995 e 2005. RevBrasEpidemiol. 2007;10(3):361-9.

3. Geue C, Briggs A, Lewsey J, Lorgelly P. Population ageing and healthcare expenditure projections: new evidence from a time to death approach. Eur J Health Econ. 2014;15(8):88596.

4. Beard JR, Bloom DE. Towards a comprehensive public health response to population ageing. Lancet. 2015;385(9968):658-61.

5. Instituto Brasileiro de Geografia e Estatística (IBGE). Pesquisa nacional por amostra de domicílios. síntese de indicadores sociais, $2015 . \quad$ Disponível em: http://ndonline.com.br/uploads/global/materias/ 2015/12/04-12-2015-02-58-43-pesquisa-ibge . pdf. Acesso em: 18 jul. 2016.

6. Paim J, Travassos C, Almeida C, Bahia L, Macinko J. The brazilian health system: history, advances, and challenges. Lancet. 2011;377(9779):1778-97.

7. Pucca Jr GA, Gabriel M, de Araujo ME, de Almeida FC. Ten years of a national oral health policy in Brazil: innovation, boldness, and numerous challenges. $J$ Dent Res. 2015;94(10):13337.

8. Guimarães MRC, Pinto RS, Amaral JHL, Vargas AMD. Desafios para a oferta de prótese dentária na rede de saúde pública. Rev Odontol UNESP. 2017;46(1):39-44.

9. Colussi CF, Patel FS. Uso e necessidade de prótese dentária no Brasil: avanços, perspectivas e desafios. Sau\&Transf Soc. 2016;7(1):41-8.

10. Frenk J. Reinventing primary health care: the need for systems integration. Lancet. 2009;374(9684):170-3.

11. Reis CMR, Matta-Machado ATG, Amaral JHL, Werneck MAF, Abreu MHNG. Describing the primary care actions of oral health teams in Brazil. Int J Environ Res Public Health. 2015;12(1):667-78.

12. Macêdo MSR, Chaves SCL, Fernandes ALC. Investments and costs of oral health care. Rev Saúde Pública. 2016;50:41.

13. World Bank. Becoming old in an older Brazil. Washington DC: World Bank; 2011

14. Thomson WM. Social inequality in oral health. Community Dent Oral Epidemiol 2012; 40 Suppl 2:28-32.

15. An JY, Darveau R, Kaeberlein M. Oral health in geroscience: animal models and the aging oral cavity. Geroscience. 2018;40(1):1-10.

16. Murray Thomson W. Epidemiology of oral health conditions in older people. Gerodontology. 2014;31(Suppl1):9-16.

17. Thorstensson $H$, Johansson $B$. Why do some people lose teeth across their lifespan whereas others retain a functional dentition into very old age? Gerodontology 2010; 27(1):19-25.

18. Neri AL, Yassuda MS, Araújo LF, Eulálio MC, Cabral BE, Siqueira MEC, et al. Metodologia e perfil sociodemográfico, cognitivo e de fragilidade de idosos comunitários de sete cidades brasileiras: Estudo FIBRA. Cad Saúde Pública. 2013; 29(4):778-92.

19. Wickrama K, Mancini JA, Kwag K, Kwon J. Heterogeneity in multidimensional health trajectories of late old years and socioeconomic stratification: a latent trajectory class analysis. J Gerontol B Psychol Sci Soc Sci. 2013; 68(2):290-7.

20. Petersen PE, Kandelman D, Arpin S, Ogawa H. Global oral health of older people- call for public health action. Community Dent Health. 2010;27(4 Suppl 2):257-67.

21.21- Ferreira RC, Vargas AMD, Fernandes NCN, Souza JGS, Sá MAB, Oliveira LFB, et al. O idoso com comprometimento cognitivo apresenta pior condição de saúde bucal? Ciênc Saúde Colet. 2014;19(8):3417-28.

22.22- Marcus SE, Drury TF, Brown LJ, Zion GR. Tooth retention and tooth loss in the permanent dentition of adults: United States, 1988-1991. J Dent Res. 1996;75(Spec No):684-95.

23.23- Imholz B, Combescure C, Scolozzi P. Is age of the patient an independent predictor influencing the management of cranio-maxillofacial trauma? A retrospective study of 308 patients. Oral Surg Oral Med Oral Pathol Oral Radiol.2014;117(6):690-96.

24. Ando A, Ohsawa M, Yaegashi Y, Sakata K, 
Tanno K, Onoda T, et al. Factors related to tooth loss among community-dwelling middleaged and elderly Japanese men. J Epidemiol 2013;23(4):301-6.

25. Wu B, Hybels C, Liang J, Landerman L, Plassman B. Social stratifcation and tooth loss among middle-aged and older Americans from 1988 to 2004. Community Dent Oral Epidemiol 2014;42(6):495-502.

26. Peres MA, Barbato PR, Reis SC, Freitas $\mathrm{CH}$, Antunes JL. Tooth loss in Brazil: analysis of the 2010 Brazilian Oral Health Survey. Rev Saúde Pública. 2013;47(suppl. 3):78-89.

27. Nascimento $S$, Frazão $P$, Bousquat $A$, Antunes JLF. Condições dentarias entre adultos brasileiros de 1986 a 2010. Rev Saúde Pública. 2013;47(suppl. 3):69-77.

28. Bernabé E, Sheiham A. Tooth loss in the United Kingdom--trends in social inequalities: an ageperiod-and-cohort analysis. PLoS One. 2014; 9(8):e104808

29. Geib LTC. Determinantes sociais da saúde do idoso. Ciênc Saúde Coletiva. 2012;17(1): 123-33.

30. Soares MRP, Istoe RSC. Alfabetização e inclusão de pessoas idosas: uma proposta interdisciplinar mediada pelas tecnologias da informação e da comunicação. Rev Cient Interd. 2015;2(3):165-75.

31. Dutra CESV, Sanchez HF. Organização da atenção á saúde bucal prestada ao idoso nas equipes de saúde da Estratégia Saúde da Família. Rev Bras Geriatr Gerontol. 2015: 179-88.

\section{CONFLITO DE INTERESSES}

Os autores declaram não haver conflitos de interesse

AUTOR PARA CORRESPONDÊNCIA

Maria Helena Chaves de Vasconcelos Catão

Universidade Estadual da Paraíba- UEPB,

58401- 490 Campina Grande- PB, Brasil

E-mail: mhelenact@zipmail.com.br 